\title{
Medication Dispenser
}

National Cancer Institute

\section{Source}

National Cancer Institute. Medication Dispenser. NCI Thesaurus. Code C128539.

Healthcare providers who dispense or administer the drug, settings in which the drug is dispensed or administered, or authorized representatives of the setting in which the drug is dispensed or administered. This encompasses a wide range of settings, including outpatient pharmacies, chain pharmacies, independent pharmacies, mail-order pharmacies, clinics, infusion centers, hospitals, health systems, and closed health systems. 